Classification

Physics Abstracts

$64.70 \mathrm{~J}-68.45$

\title{
Observation of an anomalous adsorption in a critical binary mixture
}

\author{
D. Beysens and S. Leibler \\ S.P.S.R.M., Orme des Merisiers, 91191 Gif sur Yvette Cedex, France
}

(Reçu le 23 novembre 1981, accepté le 14 janvier 1982)

\begin{abstract}
Résumé. - Nous avons démontré expérimentalement l'existence d'une nouvelle anomalie critique dans un mélange binaire, due à l'adsorption préférentielle d'un des composants par une paroi solide.
\end{abstract}

Abstract. - We have experimentally demonstrated the existence of a new critical anomaly in a binary mixture, due to the preferential adsorption of one of the components onto a solid wall.

1. Interaction of a binary mixture with a surface. - A mixture of two fluids which exhibits a critical phase separation point (at the temperature $T_{\mathrm{c}}$ and concentration $\phi_{\mathrm{c}}$ ) is an excellent experimental system for studying second order transitions [1]. It belongs to the same universality class as the 3-d Ising model. In particular, the order parameter fluctuations [the relative concentration $\left.\left(\phi-\phi_{c}\right)\right]$ extend over a distance $\xi$ which diverges at the transition as $\left(T-T_{c}\right)^{-v}$, with $v=0.63$.

M. E. Fisher and P. G. de Gennes recently studied [2] the interaction of such a system with a solid wall, where one of the two components $(\mathrm{N})$ is preferentially adsorbed. They were able to show that, as a function of the distance $z$ from the wall, the concentration profile $\phi_{N}$ of $N$ behaves as follows :

(i) for $z \simeq a_{0}$, a typical molecular distance, a normal adsorption phenomenon ("proximal » regime) occurs, which depends on the chemical nature of the system,

(ii) for $z>a_{0}$, a universal behaviour is observed : $\phi_{\mathrm{N}}-\phi_{\mathrm{N}}^{\mathrm{M}}=\phi_{\mathrm{N}}^{0} S(z, \xi)$, where, at $T=T_{\mathrm{c}}$, the profile decreases as $z^{-\beta / v}$ while $\beta=0.325$ is the order parameter exponent and $\phi_{\mathrm{N}}^{\mathrm{M}}$ is the bulk concentration. At $T \neq T_{\mathrm{c}}$, this slow decrease is replaced for large $z$ by the exponential variation $\exp (-z / \xi)($ Fig. 1).

We present here experimental evidence for this new critical phenomenon.

2. Experimental set up. - We have used the fluorescence of one of the components induced by a wave which is both guided and damped by the refractive index gradient. The fluorescence intensity, being proportional to the number of irradiated molecules, allows the concentration perturbations due to the wall to be measured.

We illuminated, at a large incident angle $i$, the wall of a fused silica cell containing the binary mixture, by means of a few milliwatt vertically polarized argon ion laser (L) $\left(\lambda_{0}=4545 \AA\right)$ 


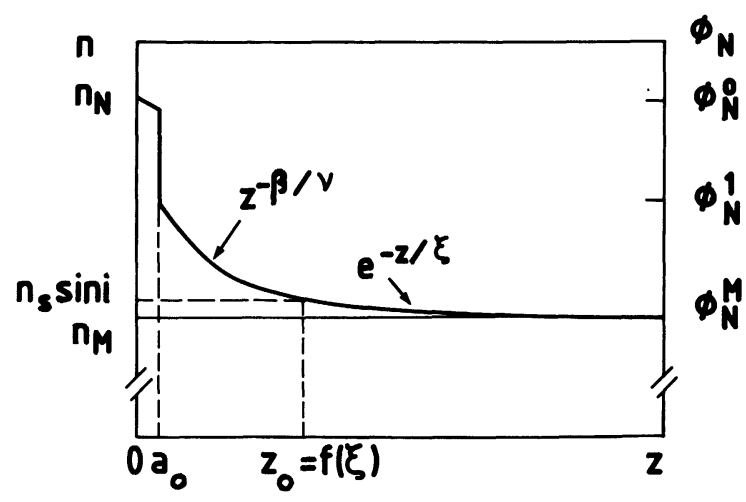

Fig. 1. - Concentration $\phi$ and refractive index $n$ profile (notations : see text) at the distance $z$ from the wall.

(Fig. 2). The scattered light is collected by a lens $(\mathrm{O})$, then focused onto a photomultiplier (P-M), after passing through a pin-hole $(\mathrm{T})$ and an interference filter $(\mathrm{F})$. The photocurrent is measured by an electrometer and calibrated by the input power determined by a photodiode $\left(\mathrm{P}_{3}\right)$. The relative accuracy is typically about $10^{-2}$. A subsidiary beam (a), monitored by the photodiode $\mathrm{P}_{2}$ and switched off during the fluorescence measurements, enables the turbidity of the system to be measured in order to determine $T_{\mathrm{c}}$. The wall under study is vertical, and the measurement point is located near the middle of the cell wall in order to avoid the gravity effects.

A mixture of Nitrobenzene $(\mathrm{N})$ and $\mathrm{n}$-Hexane $(\mathrm{H})$ has been used ; it was prepared at the critical concentration (51\% mass fraction of $\mathrm{N}$ [3]) in a cylindrical cell (C) made of highly pure silica (to avoid the self fluorescence of the wall), which was sealed under vacuum. This cell was immersed in a thermostatic water bath (B), whose temperature, stable to within $\pm 10^{-3} \mathrm{~K}$, is measured by a quartz thermometer (TH). We worked in the one-phase region, i.e. for $T>T_{\mathrm{c}}=20^{\circ} \mathrm{C}$.

We chose the $\mathrm{N}-\mathrm{H}$ system for 3 main reasons :

(i) The N-molecules are highly adsorbed onto silica [4].

(ii) $\mathrm{N}$ is highly fluorescent in the red, whereas the $\mathrm{H}$ fluorescence is located in the green, with an intensity which is lower by some orders of magnitude; using an interference filter centred at $6380 \AA$ with a bandwidth of $90 \AA$, it is therefore possible to measure the fluorescence of the $\mathrm{N}$-molecules only.

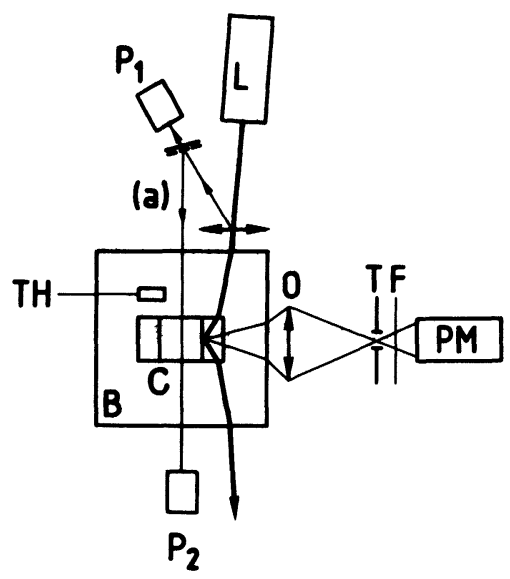

Fig. 2. - Optical arrangement. 
(iii) At $T_{\mathrm{c}} \simeq 20^{\circ} \mathrm{C}$ and for $\lambda_{0}=4545 \AA$, the refractive index of silica $\left(n_{\mathrm{s}}=1.4654\right.$ [5]) is lower than the $\mathrm{N}$ index $\left(n_{\mathrm{N}}=1.5823\right.$ [5]), but higher than the $\mathrm{H}$ index $\left(n_{\mathrm{H}}=1.3817\right.$ [5]) and only slightly exceeds the mixture index $\left(n_{\mathrm{M}}=1.4578\right.$ [6]). Since the refractive index profile $n(z)$ follows the $\phi_{\mathrm{N}}(z)$ profile, these conditions amplify the effect:

- In the case where only the proximal profile exists, it would affect only slightly the penetration depth $\delta$ and the electric field amplitude of a damped wave. These quantities are chiefly determined by the $n_{\mathrm{S}}$ and $n_{\mathrm{M}}$ indices. Since they vary slowly with $T$ (even taking into account the $n_{\mathrm{M}}$ anomaly in $\left(T-T_{\mathrm{c}}\right)^{1-\alpha}$ [7], where $\alpha \simeq 0.11$ is the specific heat exponent), no critical divergence could be detected with such a method.

- On the contrary, the existence of a long range profile, $S(z, \xi)$, should greatly increase the path of the light on the medium, the wave being transmitted as long as $z \leqslant z_{0}$ (where $\left.n_{\mathrm{N}}\left(z_{0}\right) \simeq n_{\mathrm{S}} \cdot \sin i\right)$, then completely reflected. Therefore $z_{0} \gg \delta$, and any modifications of the $\phi_{\mathrm{N}}$ profile near $T_{\mathrm{c}}$ will have striking consequences, both on the dimensions of the illuminated volume and the electric field intensity, considerably changing the fluorescence intensity.

3. Results. - We first verified (i) that multiple scattering did not affect the measurements, (ii) that when the laser beam was refracted, both the fluorescence intensity $I_{\mathrm{i}}$ at the point of incidence on the wall-iiquidi interface and the bulk intensity $I_{\mathrm{v}}[8]$ did not exhibit a critical behaviour (Fig. 3); indeed, for $T-T_{\mathrm{c}} \lesssim 0.1 \mathrm{~K}$ where the thermal variations of the $n_{\mathrm{S}}, n_{\mathrm{N}}$ and $n_{\mathrm{M}}$ indices can be neglected, $I_{\mathrm{i}}$ and $I_{\mathrm{v}}$ do not vary with temperature [9].

We have then measured $I_{\mathrm{e}}$, the intensity of fluorescence close to the wall induced by a guided wave in the refractive index gradient $n(z)\left(i \simeq 85^{\circ}\right.$ in silica). For $T-T_{\mathrm{c}} \gtrsim 0.1 \mathrm{~K}, I_{\mathrm{e}}$ behaves as $I_{\mathrm{i}}$ because of the thermal variations of the refractive indices; in contrast, closer to $T_{\mathrm{c}}$, we observed an anomalous behaviour for $I_{\mathrm{e}}$. On figure 3 (with logarithmic scales), it is clear that the $I_{\mathrm{e}}$ divergence near $T_{\mathrm{c}}$ may be well described by the power law $I_{\mathrm{e}} \sim\left(T-T_{\mathrm{c}}\right)^{-x}$, where $x \simeq 0.15$. This exponent cannot be easily related to the other critical exponents since we do not know the exact concentration profile $S(z, \xi)$, and also because of the complexity of the optical system.

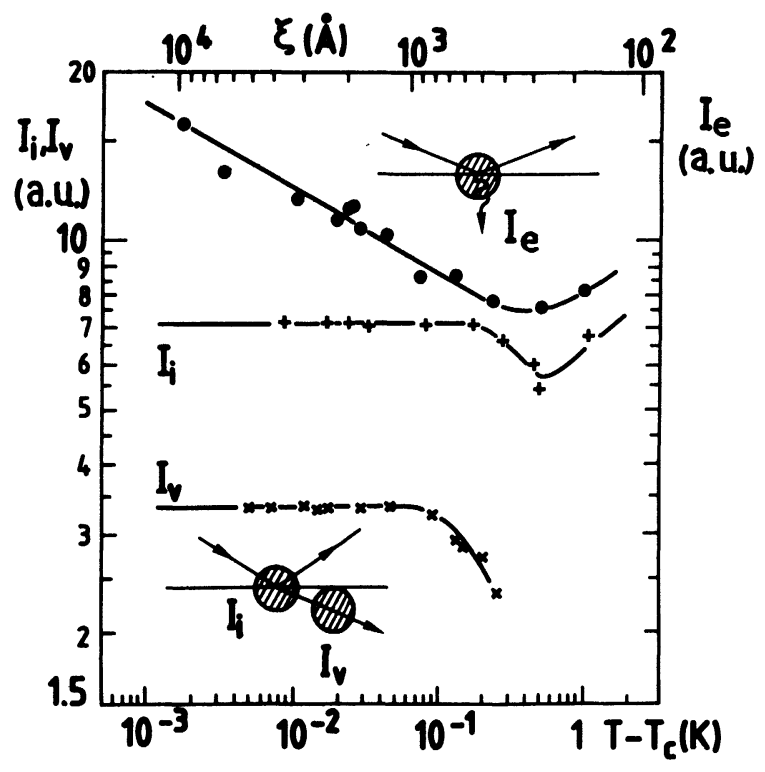

Fig. 3. - Variations versus $T-T_{\mathrm{c}}$ or the correlation length, $\xi$, of the fluoresced intensities, $I_{\mathrm{v}}(\times):$ bulk ; $I_{\mathrm{i}}(+)$ : bulk at the point of incidence; $I_{\mathrm{e}}(\bullet)$ : due to a damped-guided wave (log-log plot). 
4. Conclusion. - We have experimentally demonstrated the existence of a new critical phenomenon in a binary mixture; this phenomenon is due to the critical adsorption of one of the components onto a solid wall.

With a higher wall refractive index, one can measure the fluorescence induced by a single damped wave. By varying the incidence angle $i$, and therefore the penetration depth $\delta$, we will be able to determine, as did C. Allain for polymer solutions [10], the concentration profile $\phi_{\mathrm{N}}$ and its temperature variation. This experiment is underway.

Acknowledgments. - We are grateful to C. Allain, P. G. de Gennes, D. Rivière, M. Devoret and D. Estève for fruitful discussions.

\section{References and notes}

[1] For review, see Phase Transition : Status of the Experimental and Theoretical Situation, Cargèse (1980), ed. by L. Levy, J. C. Le Guillou and J. Zinn-Justin (Plenum Press) 1981.

[2] Fisher, M. E. and DE GenNes, P. G., C.R. Hebd. Séan. Acad. Sci. Paris 287B (1978) 207.

[3] Timmermanns, J., in Physico-Chemical Constants of Pure Organic Compounds (Elsevier, Amsterdam) 1965.

[4] COOPER, D. E., Ph. D. Thesis, M.I.T. (1980) unpublished.

[5] LANDOlt-BöRnsteIn, in Eigenshaften der Materie - 8 Teil - Optische Konstanten (Berlin, Springer Verlag) 1962.

[6] Experimental value of reference [7] extrapolated at $4545 \AA$.

[7] ZALCZER, G. and BeYSENS, D., preprint. See also :

Beysens, D. and Bourgou, A., Phys. Rev. A 19 (1979) 2407 and Proceedings of the 8th Symposium on Thermophysical Properties; Gaithesburg, U.S.A., 1981 (in Press).

[8] The path of light in the mixture is very short in order to avoid turbidity effects. See Puglielli, V. and Ford, N., Phys. Rev. Lett. 25 (1970) 143.

[9] It is interesting to note here that the surface effect $I_{\mathrm{s}}=I_{\mathrm{i}}-I_{\mathrm{v}}$, which is expected to be negligible when comparing both illuminated volumes, is found to be of the same order of magnitude as $I_{\mathrm{v}}\left(I_{\mathrm{i}} \simeq 2 I_{\mathrm{v}}\right)$. This strong surface fluorescence is hardly visible in pure $\mathrm{N}$, but appears in dilute $\mathrm{H}$ solutions. In a freshly prepared cell of $\mathrm{N}+\mathrm{H}$ at the critical concentration, $I_{\mathrm{s}}$ increases with time to reach a constant value (time constant $\tau \simeq 60 \mathrm{~min}$.). These observations identify $I_{\mathrm{s}}$ as the strong fluorescence of the proximal $\mathrm{N}$ layer, amplified by the adsorption of impurities, and eventually by the wall itself.

[10] Allain, C., private communication. 